\title{
El Abordaje de la Problemática de EXPLOTACión SEXuAL INFANTIL EN CARTAgenA ${ }^{*}$
}

\author{
Por: Mabel Valencia Mosquera \\ Carlos Ospina Bozzi
}

\begin{abstract}
Cuando el cincel esculpa los surcos de tu cuerpo herido Cuando niña mujer libre y digna seas Cuando redimir tu sexo puedas
\end{abstract}

\section{RESUMEN}

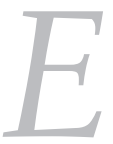

ste documento muestra el contexto en el cual se está desarrollando en Cartagena la problemática de la explotación sexual infantil, los estudios e intervenciones que se han venido adelantando para buscar solución a la problemática. De otra parte presenta apartes de una propuesta educativa que apunta a la responsabilidad social que la ciudadanía, entes gubernamentales y ONG's deben asumir en la erradicación del fenómeno.

\section{Niños y Jóvenes en Medio de las Transformaciones que Sufre la Ciudad}

En la última década Cartagena ha sido escenario de un conjunto de transformaciones en todos los ámbitos de su vida social, tanto como consecuencia del orden nacional como en respuesta a condiciones externas ligadas a aspectos globales.

Algunas de estas transformaciones han agudizado las condiciones de pobreza y miseria, de desempleo, de informalidad, subempleo, corrupción y criminalidad, situaciones que propician con frecuencia en los hogares el ausentismo de los padres, la violencia intrafamiliar y en la población menor de edad: la deserción escolar, el trabajo infantil y el fenómeno de los niños y niñas de la calle. El incremento del fenómeno de la explotación sexual de niñas, niños y adolescentes está ligado a estas y otras condiciones, y constituye uno de los efectos más corrosivos de tales transformaciones para la estructura social.

* Artículo producto del proyecto de investigación titulado: "Explotación Sexual Infantil en el Distrito de Cartagena de Indias año 2005, Facultad de Ciencias Sociales y Educación, Universidad de Cartagena. Otros miembros del equipo de investigación: Milton En Cartagena, al igual que en otras ciudades, persisten rezagos socioculturales referidos a las relaciones diferenciales según el género, la clase social, o el grupo Cabrera Fernández, Alix Pacheco Turizo y Germán Danilo Hernández Torres. 
étnico al que se pertenece, tipos de relación que a su vez modelan las identidades de hombres, mujeres, niños-niñas, ricos y pobres favoreciendo la dominación masculina, la explotación y el poder, y en correspondencia con ello la sumisión de las mujeres, los niños y niñas. Este contexto sirve de caldo de cultivo para el incremento de la violencia y explotación sexual, que precisamente se da en mayor medida hacia las mujeres, muchas de ellas adolescentes y aún niñas, mientras que el resto de la sociedad se muestra indiferente o tiende a ocultar el problema.

Cartagena declarada por la UNESCO Patrimonio Histórico y Cultural de la Humanidad cuenta con diversos atractivos de tipo arquitectónico, como sus murallas, estructuras coloniales, militares, monumentos religiosos, diversos sitios de interés histórico-cultural; con una importante extensión de playas y una serie de islas cercanas a las mismas; con una moderna infraestructura para cubrimiento de eventos, además de una diversidad de restaurantes, discotecas, y otros espacios de esparcimiento. Es considerado el más importante centro turístico de la costa caribe colombiana. De ahí que se vea visitada por un creciente flujo de turistas, lo que lastimosamente ha conllevado también al aumento de la explotación de niños(as) y adolescentes.

"No son de aquí, eran monos, altos...todos los días íbamos y nos pagaban $\$ 5.000$ los viernes...habían también colombianos, gente de caché bien elegantes, estaban pendientes que uno no soltara la lengua, nos amenazaban que nos iba a matar..." niña de 13 años).

"son extranjeros. Los extranjeros que vienen a los restaurantes vienen buscando a peladas así para sexo" (niña informante de 15 años).

Dada la vocación turística de la ciudad, la problemática reviste una especial complejidad. La evidente pauperización de la población, el crecimiento acelerado por el desplazamiento forzado, el incremento de la violencia en todas sus formas y la constitución de nuevos símbolos de consumo, de estatus y representación juvenil, son aspectos que aportan al desarrollo de la explotación sexual. Sin lugar a dudas, durante la última década, el negocio ha llegado a tocar a casi todos los sectores de la ciudad, a todos los estratos sociales y a muchas instituciones; no obstante, también se reconoce que más allá del crecimiento del fenómeno, durante los últimos años se ha dado un proceso de visibilización del mismo, en el que tienen incidencia los esfuerzos de integración institucional adelantados.

Por esta razón, desde hace algunos años se ha venido buscando la constitución de un frente Inter-institucional para recabar los esfuerzos de información sobre 
el problema, adelantar procesos conjuntos de investigación y estar entonces, en capacidad de definir conceptual y operativamente estrategias de prevención e intervención.

Entre estas iniciativas, una de las más importantes la constituye la investigación liderada por el Instituto Colombiano de Bienestar Familiar, ICBF, en donde la Universidad de Cartagena - Facultad de Ciencias Sociales y Educación participó como entidad asesora.

Este estudio confirmó la aparición de distintos actores y novedosos medios para adelantar el comercio sexual de menores, involucrando personal administrativo del turismo, de discotecas, bares y centros nocturnos, así como a comerciantes formales e informales ubicados en espacios públicos, entre otros.

\section{Factores Asociados al Incremento de la Explotación Sexual Infantil en Cartagena}

Estudios realizados en la ciudad por la Fundación Renacer (1.996), y por ICBF, Universidad de Cartagena y Renacer (2.005) apuntan a señalar factores asociados, múltiples y variados, que contribuyen directa o indirectamente a la explotación sexual y que se modifican de acuerdo con la dinámica de la misma y el contexto en el que esta aparece. Para la erradicación del problema lo más importante es poder llegar a entender estos factores, hacerles seguimiento y encontrar el camino para incidir en ellos aplacándolos, transformándolos o atacándolos.

En este sentido, considerar, en principio el comportamiento situacional de algunos de algunos de estos factores asociados, resulta un insumo de gran utilidad tanto para comprender la dinámica social y el impacto de determinadas problemáticas sobre la niñez temprana y la adolescencia, como para la toma de decisiones por parte del gobierno y otras entidades interesadas en la niñez desamparada y cuyo objetivo sea definir políticas que se reflejen directamente en esta población o apoyar a las instituciones que ofrecen servicios para garantizar los derechos de los niños y niñas y desarrollar programas de prevención de sus situaciones de riesgo.

${ }^{1}$ El Banco de la República, el Observatorio del Caribe Colombiano, la Cámara de Comercio de Cartagena

En Cartagena la pobreza está asociada a carencia de bienes y servicios básicos, desplazamiento forzado de la población, deficiencias en salud, en educación, vivienda y seguridad. ${ }^{1}$ Según el Plan Maestro 2.003-2.011 la Línea de Pobreza en Cartagena fue del 63\% para el año 2.002 y el 70\% de la población ocupada sólo gana entre 1 y 2 salarios mínimos.

y La Universidad Jorge Tadeo Lozano - Seccional del Caribe. Indicadores Sociales de Cartagena. Primer semestre del 2.001. Cuadernos de Coyuntura Social. Cartagena 2.001 
${ }^{2}$ Extractos de testimonios de niños y niñas entrevistadas durante la realización del estudio "Caracterización de la Explotación Sexual en Niños, Niñas y Adolescentes en la ciudad de Cartagena. 2.004" autoras: Correa Aguilar Tania, Morales Urchela Cecilia, Turán Periñan Teresa, Peña Bibiana. Auspiciado por ICBF con la asesoría de la U. De Cartagena-Facultad de Ciencias Sociales y Educación-docentes: Rueda de Alvarado Zoraida, Hernández García Javier, Valencia Mosquera Mabel Concepción- y el apoyo de La Fundación Renacer.

${ }^{3}$ Indicadores Sociales de Cartagena. Primer semestre de 2.004. Cuadernos de Coyuntura Social. Abril de 2.005. la Universidad Jorge Tadeo Lozano, Seccional del Caribe, el Banco de la República, Observatorio del Carib Colombiano y la Cámara de Comercio de Cartagena Revista de Coyuntura Social. No 8.Págs. $10-13$.

${ }^{4}$ Indicadores Sociales de Cartagena. Op cit. Pág 19
La pobreza juega un papel clave en la explotación sexual. Mientras la satisfacción de las necesidades básicas es apremiante, las opciones laborales son escasas lo que obliga a las familias de los sectores populares más pobres a que todos sus miembros contribuyan al sostenimiento buscando cualquier tipo de trabajo que genere algún ingreso, aunque ello arriesgue a niños y niñas a ser sometidos a diferentes formas de explotación.

"mi mamá ya no está trabajando y ella lo que consigue es pa! la comida, yo quisiera trabajar fijo porque yo quisiera ayudarla a ella y también a mí hija pero como no consigo trabajo, me toca hacer lo que pueda..." (niña de18 años). ${ }^{2}$

"Yo no había desayunado...Me dijo: Yo te doy este billete y tu... cu, ¿culeas? conmigo, entonces dije no y entonces dijo: tú ya desayunaste y yo le dije no, bueno te doy desayuno y te doy este,... como yo tenia hambre yo le dije que sí y entonces casi todos los días él me llamaba porque casi todos los días mi mamá no me daba desayuno entonces él me daba billete..." (niña de 9 años)

En Cartagena, a Octubre del 2.003, el Ministerio de Protección Social -Seccional Cartagena, había otorgado 692 permisos a menores de edad de las 752 solicitudes recibidas, para trabajar en diferentes actividades especialmente supermercados y almacenes de cadena, sin embargo, hay un buen número de niños que realizan trabajo callejero (ventas ambulantes, ayudantes de mecánica, en medios de transporte, lavadores de carros, carretilleros en plazas de mercado), de los que no se conoce una cifra exacta, aunque se presume que puedan ser alrededor de $15.000 .^{3}$

“...yo antes estaba trabajando donde mi tía pero es que ella me tenía de esclava, me veía ocupada haciendo algún oficio y me ponía otro y yo me cansé, los dedos de las manos se me comieron" (niña de 16 años).

"y yo dije no joda mi mamá no me da comida viendo que yo soy su hija mayor, pero todo bien yo voy a buscar donde trabajar donde sea, un día le dije a una amiga mía este... hay están buscando un esparri vamos a esparriar hay en el bus de Lemaitre Mercado" (niña de 15 años).

Cartagena, es una zona preferencial de alta recepción de desplazados; según la Red de Solidaridad Social - Seccional Bolívar, a mayo de 2.004, habían 7.545 familias desplazadas residentes en Cartagena distribuidas en diferentes barrios de la ciudad La presencia de desplazados en la ciudad ha agudizado problemas tales como inseguridad, la falta de servicios públicos, la informalidad laboral y la ocupación de terrenos no aptos para la construcción de vivienda, ni garantes de la seguridad de sus pobladores. ${ }^{4}$ 
Una de las consecuencias de la violencia y el desplazamiento es la pérdida de tierra y el desarraigo, situación que acarrea un rápido proceso de desorganización familiar, debido al cambio abrupto de responsabilidades y la dificultad para satisfacer sus necesidades básicas. Las principales víctimas de este fenómeno son los niños y niñas, a quienes frente al requerimiento de sobrevivencia física, le son ignoradas sus necesidades emocionales y deben entrar a cubrir responsabilidades de sostenimiento familiar con las consecuencias y riesgos de violencia y explotación señalados.

No es raro el padecimiento de enfermedades de transmisión sexual, embarazo precoz e indeseado, abortos provocados y no asistidos, lesiones físicas, deficiencia en el crecimiento (por mala alimentación, pérdida de sueño y peso), dependencia química, entre otras; todo lo cual puede comprometer el desarrollo de una sexualidad normal, sana y perpetuar la explotación sexual. ${ }^{5}$

"yo estuve embarazada y me lo saqué con unas pastillas, mí mamá me las compró y me las hizo da, pero yo no lo quería tener," (niña de 15 años).

"por aquí no se ve nada de eso" (respuesta a la pregunta: Se ha adelantado algún programa de prevención para jóvenes en relación con la sexualidad?

Según estadísticas del ICBF - Regional Bolívar en el 2.004 se presentaron 1.628 denuncias por maltrato infantil. ${ }^{6}$ Entre las consecuencias del maltrato o de la exposición a la violencia intrafamiliar está el deterioro de salud física y mental. La problemática suele agudizarse cuando los vínculos afectivos con otros miembros de la familia se van perdiendo, y la niña o el niño terminan siendo excluidos de la misma; si además, como suele suceder en estos casos, es excluidoexcluida del sistema escolar por bajo rendimiento, lo que le imposibilita alcanzar las competencias y el conocimiento necesarios para buscar mejores alternativas de desempeño ocupacional, se va viendo empujado-da bien al fenómeno de la callejización, donde se verá en mayor riesgo de ser explotada-explotado, o bien a continuar directamente utilizando como instrumento de sobrevivencia su cuerpo.

"él desde chiquitica siempre me ha humillado, quería pegarme por la cara, siempre pegarme por la cara y mi mamá no decía na". (niña de 15 años).

"A veces me acuerdo cuando mi mamá vivía con mi papá que pasaban peleando y que yo nunca me críe con ellos, porque ellos siempre me han tratado mal a mi ". (niña de 18 años

Ante las dificultades económicas y la falta de conciencia crítica sobre su situación de explotados o explotadas, la ausencia o desconocimiento de un padre que les

\footnotetext{
${ }^{5}$ Instituto Interamericano del Niño. Op. Cit. Pág 27

${ }^{6}$ MARTÍNEZ Bermúdez Elvis. La Violencia Intrafamiliar y el Maltrato Infantil siguen siendo altos en Cartagena. El Universal. Abril 2.005
} 
permita sentirse apoyados o protegidos, el ausentismo de una madre que debe velar por el sustento, niños y niñas explotados ven a veces en sus explotadores más unas figuras de apoyo, y la solución a sus dificultades, que un riesgo a su integridad, pues estos pueden llenar de ilusiones a sus víctimas, quienes se alimentan de los sueños, producto del dinero fácil, aunque la experiencia muestra que los niveles de vida prácticamente permanecen inalterados.

El temor de ser culpabilizados, o el miedo a que las amenazas de sus explotadores se conviertan en realidad, hacen que muchas veces no compartan sus experiencias con los más allegados, silenciando su padecimiento y situación de explotación, lo que intensifica sus sentimientos de desolación.

"con él (un vecino de 50 años que vive sólo) mi mamá y yo nos sentimos agradecidas porque el me paga el colegio, me da para vestirme, me lleva y me trae del colegio, me saca a pasear los domingos ".(niña de 14 años).

"el es un niño que no es muy comunicativo por lo que usted me esta comunicando, no el nunca expresa lo que siente, no tiene esa confianza conmigo" (la madre de un niño explotado sexualmente de 17 años).

"y mi mamá también dizque me iba a echar de aquí mi padrastro me iba a mandar pa donde mi papá mi papá... ninguno de los dos me querían ninguno de los dos me querían aquí no me querían y no me querían allá". (Niño de 16 años).

"No, yo pienso que hoy uno esta aquí y conoce a una persona y uno puede que comparta con ella cosas, pero esa persona no es mi amiga". (Niña de 17 años).

"O sea me gustaría estar bien, estar trabajando, tener mis cosa por mí misma y tratar de írmela bien con mi mamá, pero no sé...(niña de 18 años).

"porque a mi hace rato se me acabaron los sueños, uno cuando se mete,. por ejemplo, los que se meten a la droga o a la prostitución a cada uno se le acaban los sueños..." (niña de 17 años).

“... la mariguana yo me la fumo y me da por despejar la mente porque la mariguana a mí me hace reír, me pongo allá a reírme con estos pelaos yo digo estoy peleando con ella y yo me voy me fumo mi tabaco y me quedo ahí, riéndome con estos pelaos..." (niña de 15 años).

Se encuentra que los niños y niñas explotadas sexualmente debido a los condicionantes desfavorables del entorno donde se desenvuelven no siempre pueden acceder al sistemas educativo formal o se encuentran atrasados frente a los demás de su misma edad; por lo que sus posibilidades de una búsqueda de consecución laboral ajustadas a una buena calidad de vida se restringen y se ven abocados-as a seguir siendo explotados(as) laboralmente. 
Usualmente, desertan entre los 11 y los 15 años y comienzan a vivir de trabajos temporales, y a veces del robo. Desertan por razones económicas: necesidad de colaborar con la familia, por falta de interés en lo que se les está enseñando o por problemas conductuales y de relación con los(as) demás. Si se abandona la escuela para iniciarse en empleos informales, que suelen requerir jornadas excesivas, pero ofrecer bajos salarios y precarias condiciones laborales, se estaría recrudeciendo la pobreza y por ende aumentando los riesgos con relación a la misma, ya contemplados

"para sorpresa mía, el profesor me informó que el niño no registraba asistencia al colegio. Sólo había asistido durante los primeros días del mes de febrero y los meses siguientes los tenía no parecía registrada la asistencia a clases" (madre de un niño de 17 años).

"no seguí estudiando porqué me salí con un muchacho y el era bandido y ahora está preso..." (niña de 15 años).

"En el colegio me decían y que tu tienes la cara como una arenca frita entonces yo tuve que echarle una mentira a mi mamá para que no me mandara mas al colegio, le dije que la profesora me había dicho que no fuera más que si no llevaba más él uniforme que no me mandara más y mi mamá no tenía plata y no me mandó más estaba haciendo cuarto y quinto para ir para sexto".

Si los gobiernos no proveen los fondos necesarios ni insertan en su agenda de la Infancia el fenómeno de la explotación sexual como de urgente consideración, entonces no cabe otra alternativa que esperar que el mismo continúe creciendo. Colombia al ratificar la Convención de los Derechos del Niño por medio de la Ley No. 12 de 1.991 adquirió la obligación de respetarla y cumplirla; y los gobiernos el compromiso de asegurar su aplicación. Tal convención reza en su artículo 34 que: "Los Estados partes se comprometen a proteger al niño contra todas las formas de explotación y abusos sexuales...". ${ }^{7}$

Colombia se cuenta entre los países de la OEA con Plan de Acción contra la Explotación sexual de niños, niñas y adolescentes. Pero además se requiere de un proceso continuo y sostenido de planificación y ejecución de tales planes de

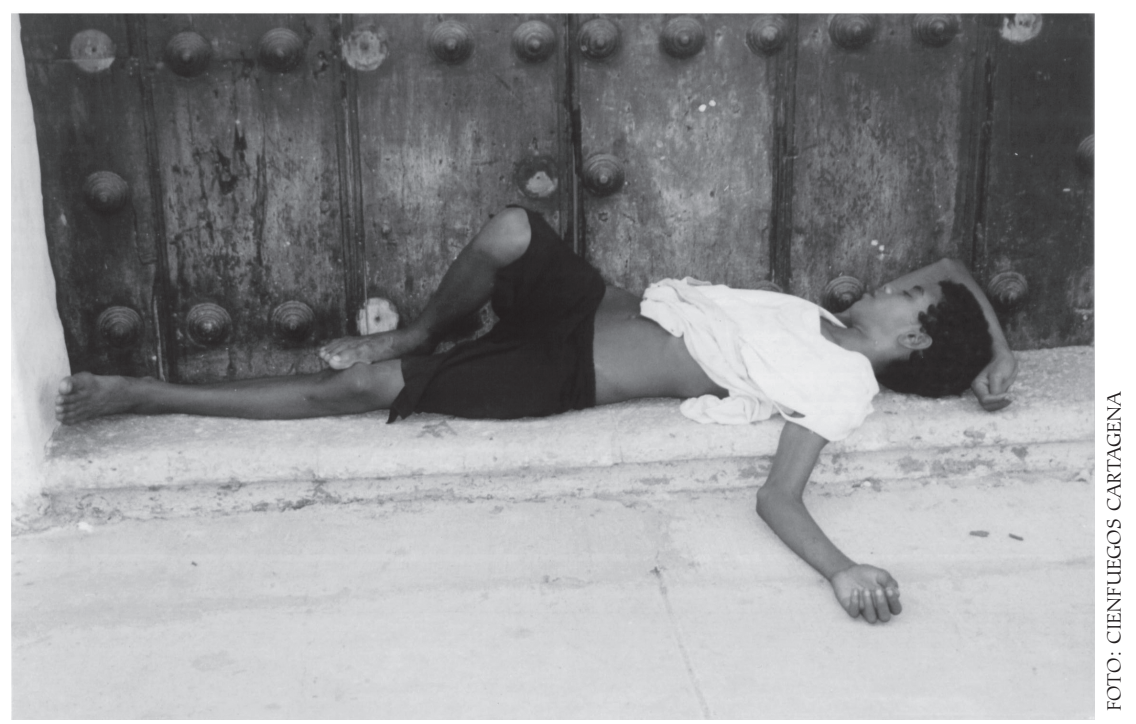


${ }^{8}$ Ibidem

9 Esta información se obtuvo del Informe y sus anexos periodísticos correspondientes: "Análisis exploratorio del cubrimiento informativo sobre la Explotación Sexual de Niños, Niñas y Adolescentes en el periódico "El Universal"período comprendido entre Enero del 2.004 y Agosto del 2.005", análisis presentado por el profesor Milton Cabrera Fernández como resultado del estudio exploratorio de fuentes periodísticas realizado por los estudiantes de IV semestre del Programa de Comunicación Social (período II-2.005) en desarrollo de la cátedra "Prensa y Manejo de Fuentes". acción y programas técnicos que vinculen a las organizaciones de estado y de la sociedad civil, identificando los grupos de riesgo, estableciendo los servicios de atención integral con criterios de focalización geográfica, institucional y de vulnerabilidad, así como capacitando a personal idóneo como operadores del sistema de protección social, o del sistema de protección judicial. ${ }^{8}$

\section{Avances de la Investigación y Gestión sobre el Tema de la Prostitución Infantil}

A pesar de los esfuerzos investigativos no se tienen datos concretos que permitan sistematizar la totalidad de casos de niños(as) y adolescentes explotados sexualmente en la ciudad de Cartagena y realizar los monitoreos necesarios para determinar su evolución. Gran parte de ello se debe a las características de clandestinidad del fenómeno, que hace difícil contactar a los actores sociales involucrados, pero también está el temor recurrente de los niños(as) y adolescentes padecientes a hablar sobre ello.

Sin embargo, y a pesar de las dificultades para consolidar estas estadísticas, los resultados del proyecto ICBF, Renacer y Universidad de Cartagena dados a conocer a la comunidad cartagenera el 16 de Marzo del 2.005 despertaron una gran inquietud en diferentes entidades sobre el fenómeno lo que condujo a que se fueran realizando diferentes eventos con el fin de sensibilizar a la población sobre la problemática y hacer visible las características y consecuencias de la misma, lo mismo que aunar esfuerzos que favorezcan la denuncia, y capacitar a la población hacia la prevención de la situación.

Los eventos adelantados contribuyen además a visibilizar la complejidad de la problemática: ${ }^{9}$

- Foro sobre Explotación Sexual Infantil y Turismo organizado por el secretariado de Pastoral Social de la Arquidiócesis de Cartagena, con el propósito de promover en los diferentes actores sociales, incluidos niñosniñas y adolescentes, en los medios de comunicación y ciudadanía en general el interés por el conocimiento, prevención y so lución a esta problemática. Hicieron presencia directores y directoras de instituciones nacionales e internacionales cuya responsabilidad es trabajar por la niñez.

- Mesas de Trabajo en las casas de Justicia de Chiquinquirá bajo la coordinación del Comité Internacional para el desarrollo de los Pueblos, la Alcaldía de Cartagena y Renacer y con la participación de líderes cívicos, profesores y funcionarios públicos quienes aportaron ideas sobre cómo luchar en contra 
del problema y hacerle frente a los principales factores asociados al mismo, tales como: la pobreza, el desplazamiento, la desintegración familiar. 18 a 20 de Mayo del 2.005.

- Lanzamiento de la Campaña "Encuentre el rostro de la Violación" por parte de la Corporación Red de Empoderamiento de las Mujeres de Cartagena y Bolívar. Mayo XX a Junio 30. Con esta campaña se buscaba brindar el apoyo que necesitan las víctimas de ese delito para denunciar a sus agresores.

- "Diplomado sobre Explotación Sexual Comercial a Menores" organizada por el Distrito con el apoyo de la ONG italiana Comité Internacional para el Desarrollo de los Pueblos (CISP) y la Fundación Renacer, iniciado el 5 de Agosto con duración de 2 meses en la Universidad Luis Amigó. Desde el cual se busca abordar temáticas como las causas y consecuencias, lo mismo que concientizar sobre la importancia de proteger, promover y respetar los derechos de los niños y niñas.

- Encuentro Internacional contra el Turismo Sexual "Cartagena abre tus ojos" del 17 al 19 de Agosto del 2.005. Se reunieron expertos en el tema que socializaron sus experiencias con la participación y compromiso de todos los sectores sociales. El encuentro tuvo 4 ejes temáticos: Caracterización del Turismo Sexual; Ética, Responsabilidad Social y Política; El papel de los Medios de Comunicación y Legislación Nacional e Internacional.

- "Simposio para la Comunidad. Violencia Sexual, Niñez y Juventud. Una perspectiva Psiquiátrica". Realizado el 13 de Octubre por la Asociación colombiana de Psiquiatría y el Instituto Colombiano de Bienestar Familiar.

Los medios de comunicación también aportan a dilucidar esta problemática, un estudio exploratorio de fuentes periodísticas realizado por los estudiantes de IV semestre del programa de Comunicación Social de la Facultad de Ciencias Sociales y Educación encontró que durante los últimos 20 meses se publicaron en el periódico El Universal 48 informaciones sobre explotación y abuso sexual; publica este medio información alusiva a casos específicos relativos a capturas de abusadores y explotadores, denuncias, reportes estadísticos periódicos, además de informes especiales y análisis periodísticos, reportes de eventos o foros sobre el particular.

El interés de editorialistas, columnistas y redactores de informes especiales, así como la dedicación de espacios privilegiados en secciones de gran nivel de lecturabilidad: Conceptos, Facetas, Sexto Sentido, Actualidad y Primera Página dan cuenta por un lado, de la importancia que el tema ha cobrado en el últimos tiempos en la ciudad y de otro, de cómo la prensa está ayudando a formar opinión sobre el problema, a concientizar a la ciudadanía de que la explotación 
${ }^{10}$ Frase extraída de las conclusiones de uno de los trabajos presentados por los estudiantes de la cátedra Prensa y Manejo de Fuentes, orientada por el profesor Milton Cabrera en IV semestre del Programa Comunicación Social sexual es un crimen condenable; convoca a participar en la denuncia y en la búsqueda de mecanismos para combatir este delito. Los resultados han comenzado a mostrarse según se ve representado en el envío de opiniones a la sección "Buzón" donde la mayor participación de la comunidad revela la preocupación creciente de la ciudadanía por un tema que hasta ahora se presentaba como prohibido.

"El abuso y la explotación sexual a menores son temas que, por la fuerza de sus efectos en la población más sensible y por la resonancia de hechos de incuestionable gravedad, apenas hoy comienzan a hacerse visibles en una ciudad que, por su condición turística y su actitud de constante rechazo a la autocrítica, los ha mantenido cómplicemente invisibles". ${ }^{10}$

La universidad como centro generador y transmisor de conocimientos, anima en sus docentes y estudiantes el interés por conocer e indagar sobre la realidad social del contexto. Diferentes facultades y en particular las de Medicina, desde la cátedra de Medicina Social; de Derecho y Ciencias Políticas, desde la cátedra de Metodología de la Investigación, y de Ciencias Sociales y Educación, desde diversas cátedras, han venido concientizando a los(as) estudiantes sobre el problema de la explotación sexual y sus consecuencias, lo mismo que asesorándoles y orientándoles en la realización de trabajos investigativos sobre este tema.

Docentes de diferentes facultades han venido desarrollando trabajos sobre el tema, demostrando un compromiso profesional con la niñez, la juventud y la mujer, y un ánimo inquebrantable en la defensa de sus derechos, entre esos docentes pueden citarse a: Carmen Escallón Góngora, Nayib Abdala Ripoll, Pilar Morad de Martínez, Nancy Bolaño Navarro, Hortensia Naizara Rodríguez.

La Facultad de Ciencias Sociales y Educación desde hace varias décadas viene trabajando en los temas de la familia, la comunidad y la población juvenil a través de la ejecución de proyectos investigativos y de gestión-intervención que son adelantados en procesos académicos con la participación de docentes y estudiantes. Concientes del problema del abuso y la explotación sexual, de la violación de los derechos humanos de los niños(as) y jóvenes, se han realizando una serie de actividades académicas en varias dimensiones y amplios sectores de la ciudad

En cuanto al fenómeno de la explotación sexual y contextos inadecuados para niños(as) y jóvenes la facultad participa en la Gestión de los siguientes proyectos: 
- Espacios de Reflexión para Fomentar el Crecimiento Personal de los y las Adolescentes de la Fundación Renacer. ${ }^{11}$ Como resultado del mismo se realizaron 57 talleres a adolescentes en riesgo de explotación sexual en la fase centro ambulatorio de la Fundación Renacer, logrando un mayor crecimiento personal, motivación y participación en relación con sus valores, autoestima y habilidades sociales.

- En el colegio Liceo de Bolívar institución de carácter oficial a la que asisten niños, niñas y adolescentes de los barrios aledaños, de estratos I, II y III. El programa de Trabajo Social adelanta un estudio sobre las causas de las dificultades comportamentales y de socialización que viene presentando la población estudiantil al interior de la institución. En el estudio participa además el equipo de Reeducación y Pedagogía.

- Asomenores, en esta institución los niños y niñas infractoras de la ley penal tienen la posibilidad de reeducarse para lograr su recuperación personal, familiar, social y académica. Las practicantes de la facultad en esta institución trabajan en los procesos de resocialización de los niños y niñas.

- Secretariado de Pastoral Social adscrito a la Arquidiócesis de Cartagena. En esta institución la practicante de Trabajo Social desarrolla el proyecto de capacitación relacionado con el tema de la Prevención de la Explotación Sexual Infantil.

- Convenio establecido con Plan Internacional. Un grupo interdisciplinario, de docentes de la Facultad ${ }^{12}$ viene desarrollando el Proyecto de Sensibilización y Formación a Padres y Madres para el Desarrollo de niños y niñas del semillero Defensores del Agua en las comunidades de Membrillal, Bayunca, Puerto Rey y Tierra Baja.

${ }^{11}$ ZAMBRANO Silvia María 2.005 con la asesoría de la profesora Zoraida Rueda de Alvarado

${ }^{12}$ Ana Pombo Gallardo, Amelia Segrera, Elda Erazo Dickson, Mabel Concepción Valencia y la trabajadora Social egresada de la Facultad Heidi Barranco cabo los grupos de docentes investigadores de la facultad, e igualmente a través de la realización de tutorías y asesorías que los mismos hacen a estudiantes de pregrado y postgrado. ${ }^{13}$

Las áreas trabajadas relacionadas con el tema de niños(as) y jóvenes en estado de riesgo son: Familia, Violencia Intrafamiliar, Pobreza, Desplazamiento,

${ }^{13}$ Trabajos de los cuales se tiene copia en la Unidad de Gestión de Información "Esther Pérez de Alvear" de la Facultad de Ciencias Sociales y Educación. Universidad de Cartagena 
Convivencia y Conflicto, entre otras. Para el caso que nos ocupa señalaremos algunos de los productos elaborados por los estudiantes de año social del programa de Trabajo Social:

$\checkmark$ Intervención del menor trabajador, abusado y explotado sexualmente.

$\checkmark$ Causas de la Explotación sexual de menores (Comunidad la Esperanza)

$\checkmark$ Fomento del crecimiento personal en los y las adolescentes explotados sexualmente.

$\checkmark$ Factores socioculturales como determinantes en la predisposición del menor trabajador a una práctica sexual temprana.

$\checkmark$ Diagnóstico e Intervención en la problemática de los niños, niñas y jóvenes habitantes de la calle.

$\checkmark$ Vinculación de las familias de las adolescentes embarazadas en el proceso de desarrollo integral.

$\checkmark$ Estrategias de Prevención de la Violencia Intrafamiliar.

$\checkmark$ Intervención en las familias desplazadas por el conflicto armado

$\checkmark$ Prevención del Maltrato Infantil

$\checkmark$ Factores de Reincidencia del Joven en Conflicto con la Ley Penal

$\checkmark$ Prácticas y Tendencias del Trabajo Infantil

$\checkmark$ Vínculos afectivos en los jóvenes con problemas de drogadicción.

$\checkmark$ Análisis de las prácticas de evaluación y conflictos en Instituciones educativas.

$\checkmark$ La familia y el barrio como proyecto escolar de integración y desarrollo en sectores populares.

$\checkmark$ Procesos de Educación a la familia desde la Afectividad. ${ }^{14}$

Desde la maestría en Estudios de Género, que ofrece la Facultad en convenio con la Universidad Nacional- Facultad de Ciencias Humanas, se busca seguir indagando sobre el tema; razones éticas llevan a indagar las causas, consecuencias y formas estratégicas de cambio para erradicar la problemática, mediante la elaboración y ejecución del proyecto:

"Procesos de Formación de las Identidades en Niñas y Adolescentes explotadas sexualmente", cuyos objetivos son:

${ }^{14}$ Los trabajos - Comprender el proceso de formación de identidad sexual desarrollado en las relacionados han sido asesorados por los docentes Ana Pombo Gallardo, Amelia Segrera, Hortensia Naizzara Rodríguez, Germán Danilo Hernández . niñas y adolescentes explotadas sexualmente

- Estudiar y analizar el desarrollo de dimensiones identitarias socioculturales - Reconocer el potencial de autonomía y empoderamiento que pudiera estar presente en las niñas y adolescentes explotadas sexualmente 


\section{Lo Que se Propone}

Ya que la visibilización del problema ha despertado el interés de diversas fuerzas sociales de abordarlo y asumirlo con mayor responsabilidad frente a las nuevas generaciones que están creciendo en medio de una crisis de identidad y de valores sin precedentes en la historia nacional, ponemos a consideración de la comunidad local regional y nacional las siguientes propuestas de acción.

- Explotación Sexual de Niños, Niñas y Adolescentes en La Ciudad de Cartagena de Indias.

Como resultado de la ejecución de este proyecto las investigadoras han propuesto algunas directrices para la intervención del fenómeno en la ciudad de Cartagena de Indias: ${ }^{15}$

$\checkmark$ Desarrollo de estrategias comunicativas para la visibilización social de la problemática de explotación sexual infantil y para generar procesos de pedagogía social que motiven cambios de actitud en la sociedad en general y particularmente en los niños(as) vulnerables, así como en padres y miembros de familia, maestros, e incluso adultos potencialmente susceptibles de entrar en la red de explotación sexual, como empresarios o como "clientes".

$\checkmark$ Campañas de difusión masiva dirigidas a despertar la conciencia de protección y respeto por nuestros niños, niñas y adolescentes.

$\checkmark$ Difusión en forma pedagógica de los derechos y las normatividades existentes con relación a la violencia intrafamiliar, el maltrato infantil, el abuso y la explotación sexual de niños, niñas y adolescentes.

$\checkmark$ Diseño de estrategias pedagógicas que orienten a familias, niños y adolescentes en el uso adecuado de las redes institucionales de atención en sus diversos niveles de intervención.

$\checkmark$ Fomento de espacios de participación de niños(as) y adolescentes, involucrando a la comunidad y a los distintos actores de la sociedad, de manera que se cree un frente común para bloquear la explotación sexual infantil.

La complejidad del problema implica una estrategia de comunicación amplia y continuada, y por lo tanto debe constituirse como Programa, que permita el diseño y desarrollo de medios y acciones dirigidos a grupos focales de la población, con lenguajes apropiados según edad, educación, estrato socio-económico, y posición en la red social de relaciones, por ejemplo, maestros y miembros de familia o de grupos primarios de referencia.

${ }^{15}$ Resumen Ejecutivo de "características de la Explotación Sexual en niños, niñas y adolescentes. Op Cit 
El Programa que se propone precisa activar los recursos con que cuenta la ciudad en términos de comunicación masiva, tanto en materia de prensa escrita, como del medio radial y televisivo. Serán convocados a cumplir con su papel el canal local de televisión por cable y la nueva estación de televisión educativa de la Universidad de Cartagena, así como en un futuro próximo la emisora de interés ciudadano cuya solicitud de funcionamiento está en manos del Ministerio de Comunicaciones. Estos medios constituyen las más valiosas herramientas para abordar un proceso continuado de comunicación social, que además, tendrá que llegar con productos comunicativos novedosos a poblaciones y grupos específicos: al interior de la familia cartagenera, en el sistema escolar y universitario, y a organizaciones juveniles que constituyen grupos primarios de influencia directa sobre nuestra población infantil y adolescente.

La Facultad de Ciencias Sociales y Educación propone para comenzar en el proceso educativo:

- Programa Continuado de Comunicación y Pedagogía Social

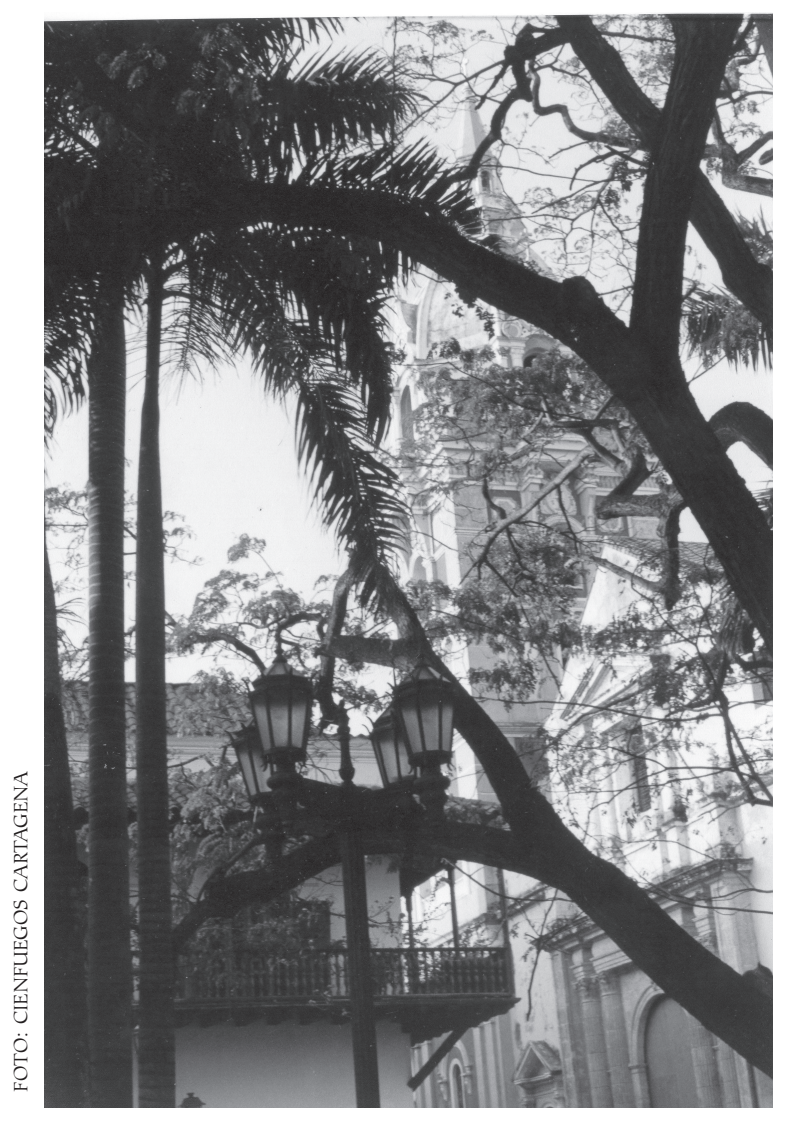

Objetivo General del Programa:

Incidir significativamente en la actitud social de la ciudadanía cartagenera, en torno a la necesidad de hacer un frente común de prevención y control del abuso y la explotación sexual de niños y adolescentes, mediante una serie de estrategias multimediadas de comunicación social, dirigidas de manera general y puntualmente a estamentos específicos de la población, entre los cuales se destacan los diversos grupos etéreos (niños y adolescentes), la familia, los y las educadoras y las instituciones de protección social.

Objetivos Específicos:

- Ofrecer a la población infantil de la ciudad información multimediada sobre la existencia de los fenómenos de abuso y explotación sexual, así como sobre sus derechos humanos y las instancias a las que puede acudir, de forma tal que al correlacionarla asuma una actitud de autocuidado y autoestima.

- Ofrecer a la población juvenil de la ciudad 
información multimediada sobre las características de los fenómenos de abuso y explotación sexual, así como sobre la integridad de su cuerpo como elemento esencial de su naturaleza humana de forma tal que al correlacionarla asuma una actitud de respeto a sí mismo, autocuidado y autoestima, que contribuya a identificar caminos diferentes a la explotación sexual.

- Ofrecer a la familia cartagenera, constituida por núcleos solidarios de parentesco, información multimediada sobre los fenómenos de abuso y explotación sexual infantil y juvenil, de forma tal que se constituya ella, mediante una actitud de vigilancia y rechazo frontal, en la primera fuerza social de prevención y control de los mismos.

Ofrecer a los educadores cartageneros -mediante estrategias multimediadasinformación y herramientas pedagógicas y psicológicas para comprender los fenómenos de abuso y explotación sexual infantil y juvenil, de forma tal que se constituyan en orientadores eficaces en torno a situaciones de riesgo, con un conocimiento y un manejo psico-social que genere confianza en niños(as) y adolescentes que ya son víctimas de los mismos.

Generar procesos de pedagogía social mediante estrategias multimediadas dirigidas a la creación de un frente de actitud social de rechazo a los fenómenos de abuso y explotación sexual infantil y juvenil, restituyendo en la cultura ciudadana los valores de la protección y la solidaridad.

Estrategia de Comunicación

El elemento central de esta estrategia radica en el carácter continuado del Programa de Comunicación Social que se diseñe, de manera tal que no se confunda con la producción puntual de una serie de materiales, sino se entienda como un proceso de formación de los distintos grupos focales involucrados y de la ciudadanía en general.

Para el efecto, el Programa estará diseñado a partir de los siguientes frentes de intervención, a manera de una trama mediante la cual se tejen diversidad de proyectos específicos, materiales y mensajes, según se identifiquen necesidades:

$\checkmark$ Identificación, caracterización y análisis de poblaciones usuarias del Programa, para determinar tratamientos adecuados de la información respecto a cada una.

$\checkmark$ Identificación, caracterización y análisis de medios apropiados según 
necesidades o sub-proyectos específicos de comunicación y pedagogía social, incluyendo la definición de estrategias de recepción individual, presencial y/ o de emisión masiva.

$\checkmark$ Identificación, caracterización y análisis de temáticas y contenidos apropiados para responder puntualmente a la complejidad de los fenómenos objeto de atención del Programa, para los diversos grupos sociales de recepción.

Marco de Referencia Conceptual Básico

Este Programa tendría como fundamento conceptual las diversas aproximaciones y desarrollos propios de la Pedagogía Social y la Comunicación para el Desarrollo como disciplinas apropiadas para un abordaje estratégico de intervención social, dirigido a incidir en actitudes y comportamientos ciudadanos. En términos de abordajes temáticos se proponen los siguientes:

$\checkmark$ Reflexión en torno al poder y los imaginarios culturales que permiten un abordaje inapropiado del mismo.

$\checkmark \quad$ El estudio del miedo proporcionará invaluables elementos para generar la ruptura de esquemas mentales que paralizan a las personas y a la sociedad en general.

$\checkmark$ Comprensión profunda del fenómeno de la incomunicación acentuado en la era globalizada, proporcionará importantes herramientas para abordar el tratamiento de la información en todos los sub-proyectos y para las distintas audiencias a las que se dirigirá el Programa.

$\checkmark$ Estudio y abordaje del conflicto entendido como diferencia y negociación cultural.

$\checkmark \quad$ El estudio de la condición humana y sus múltiples dimensiones, ofrecerá una cobertura apropiada a los demás aspectos conceptuales propuestos en este documento, así como aquellos que surjan de la discusión transdisciplinaria una vez se constituya el Programa y su equipo de trabajo.

Se requiere igualmente de un plan integrador y un ente que direccione los diferentes programas y actividades, para lo cual se propone la creación de un Centro Interinstitucional contra la Explotación Sexual Infantil en Colombia como ente de carácter nacional con sede en Cartagena de Indias, que regule todas las acciones del Estado y de entidades particulares orientadas a la prevención y control de la explotación sexual de niños, niñas y adolescentes. 


\section{BIBLIOGRAFÍA}

BANCO DE LA REPUBLICA, CÁMARA DE COMERCIO DE CARTAGENA, UNIVERSIDAD JORGE TADEO LOZANO, ANDI,, OBSERVATORIO DEL CARIBE COLOMBIANO, Indicadores Sociales de Cartagena. Primer semestre del 2.001. Cuadernos de Coyuntura Social. Cartagena. 2001

Abril de 2.005. Cuadernos de Coyuntura Social No 8 .

CABRERA, Fernández Milton. «Análisis exploratorio del cubrimiento informativo sobre la Explotación Sexual de Niños, Niñas y Adolescentes periódico "El Universal". Enero del 2.004 y Agosto del 2005

CARDENAS Stella y otros. Renacer. Una propuesta para volver a nacer. Fundación Renacer, Unicef-Colombia, Bogotá, 2000

CORREA Aguilar Tania, MORALES, Urchela Cecilia, DURAN Periñan, Teresa, PEÑA, Bibiana. "Caracterización de la Explotación Sexual en Niños, Niñas y Adolescentes en la ciudad de Cartagena. ICBF, Universidad de Cartagena, Renacer. Cartagena 2005

CORREA Aguilar Tania. Resumen Ejecutivo del Proyecto "Caracterización de la Explotación Sexual en Niños, Niñas y Adolescentes en la ciudad de Cartagena, Cartagena. Febrero, 2005

Http://News.Bbc.Co.Uk/Hi/Spanish/Science/Newsid_3545000/3545177.Stm

HTTP://WWW.PROCURADURIA.GOV.CO/HTM/EVENTOS.HTM. Edgardo Maya Villazón, Procurador General de la Nación, durante el evento "Por la Niñez y la Adolescencia, Encuentro de Gobernadores 2005

INSTITUTO INTERAMERICANO DEL NIÑO. "La Explotación Sexual en Niños, Niñas y Adolescentes en América Latina". Programa de Promoción Integral de los Derechos del Niño. 2004

MARTíNEZ Bermúdez Elvis. La Violencia Intrafamiliar y el Maltrato Infantil siguen siendo altos en Cartagena. El Universal. Abril 2.005

PINEDA Duque, Javier. Género, Masculinidad y Globalización. Ponencia presentada en el Primer congreso Internacional: Dialogo entre Civilizaciones. Iberoamérica y el Mundo Islámico. Bogotá, 2003

REGLAMENTO GENERAL DE LA PRÁCTICA ACADÉMICA. Facultad de Ciencias Sociales y Educación. Universidad de Cartagena. 1999

VALENCIA Mosquera, Mabel Concepción. Proceso de Formación de las Identidades en Niñas y Adolescentes explotadas sexualmente. Proyecto para optar al Magister en Estudios de Género. Universidad de CartagenaUniversidad Nacional, 2005

ZAMBRANO Silvia María. Gestión del Proyecto Espacios de Reflexión para fomentar el crecimiento personal de los y las adolescentes de la Fundación Renacer. 2005

\section{BIOGRAFÍA}

\section{MABEL VALENCIA MOSQUERA}

Psicóloga Universidad de Antioquia. Especialista en Docencia Universitaria Universidad Santo Tomas Cartagena, Teorías Métodos y Técnicas en Investigación Social, cursa actualmente estudio de Maestría en Estudios de Género Universidad Nacional en convenio con la Universidad de Cartagena, Docente Facultad de Ciencias Sociales y Educación.

\section{CARLOS OSPINA BOZZI}

Comunicador Social. Especialista en Administración de Programas de Desarrollo Social Universidad de Cartagena. Docente Facultad de Ciencias Sociales y Educación. 